ifademe Lou Ise-Rowan NIHBBBilL

1906 if. Appleton Street.

APPIETON - F IS CONSIN

(Stats-Unig)

\title{
Chèze Madim,
}

Hon Secretaize ext en vacances, at somm toute je le remplace, ce qui ra pe zwet tre de reponfe di rectement a ros queations:

IR- Vous ícorirez en elfat certainement pas

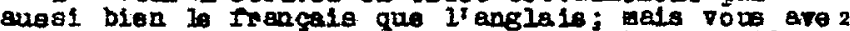
tort de dise "que vove n'e crivez pas tads gantiwent ": clest lo cont rălro.

28-On1, le $t$ in de Ia Lat to arec 1 iange. et "Le Pay cholog 18 de l'BFt" ont ét6 d6troltes paz la teatapo. Je vécris "La Paychologle de lidrt"

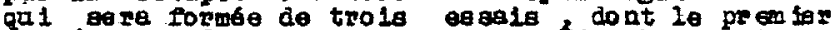
pazartza on Octobse, is aecond en treombere ot lo

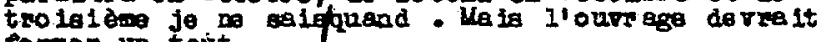
fo Fiel s un tout .

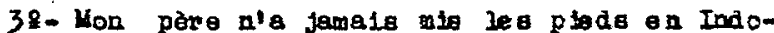
chlne. Cette lnforiation a été donnés as dout pez une Agence et complète eent fronsse. Us fanilis

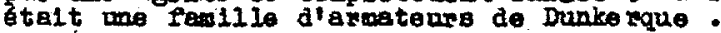

48- Bnselgnement seligleux : catholique, tomala. Fol agsez apdente jusqu' í i'adoslesconco. ensul te aǵnos tIqus.

5.8- "Prealer mriage : Blorence, 1933. Socond wage : Plerp-Gauthler at'vincent: 6 ans et 4 ans.

69- Io IIrve our Lawe onc, gu1 a'appello

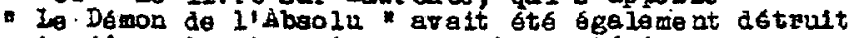

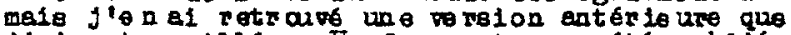

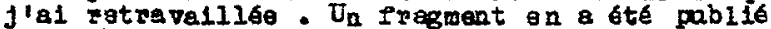
dans is se vas" Saloom nume ro de l'Hirer de cet te annes . Je le mottral sansdoute an point

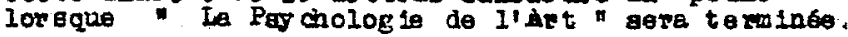




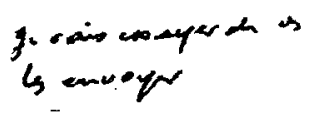

79- Vos nene lgnements anr le guerre sont exacta : j'al eté d'abord pisonnier de gagrre ot us suls oreds

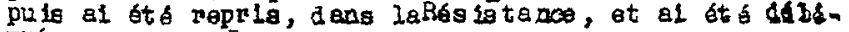
จné .

J'al foit le prouler dynentage de la Hesiatanco (Decambre 1940 ) et al ilal is guer cono Commandant dae la Beigade Alsaca-iorraine ( cietalt une unite rilitalre de comentios d'appal des chapa).

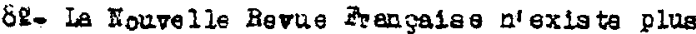
aepuis ia guoryo.

Je n'ai pablte, depuis la Liberation que deux interviows I'tune dene la Remu Gaisso"Iabys in the ot l'antre dang lo joumal "Combat. vers lo milieu - Hotsmbe 1946 et des tregments de n Ia Paychologie de liAt t que poug ietroure rez dang lo volume. I do it paraltye áx Bditlong Skira, \& Ge dève.

99- Vons arez' 2n tout qu j'al pablis d'important.

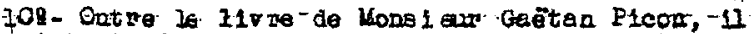
en exiate tro is or quetre autres, loins importents :

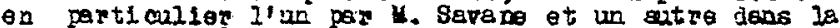
aérie belge $n$ A ls rencontio de..."

Far alliouns, un actro ilore - important olul-1a-

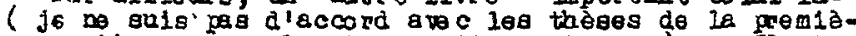
se pertio, lo cola n'a pes d'importa nos J par cleuda

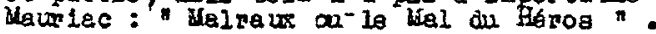

Il dolt en paraitre geno daute cette ande un eut re de Jules lonnerot, et l'année peocheine an sulvant, deno la collection "fe esenfy" des Bditiong PIOR.

Pour la guer : ci-joint lefolographio platía par I'Agence France. Fres ge loraque. je sula devenu úlais. tre de I' Information.

Volla. Aquisez-rous bign ec tous cos petita paplers.

Crojez 01 bien sjupathdigament froc vous.

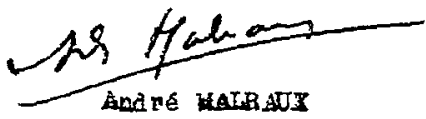




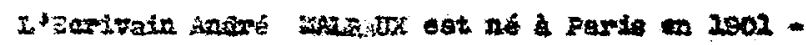

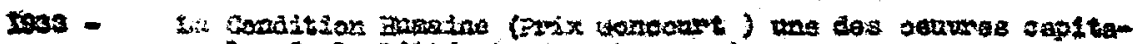

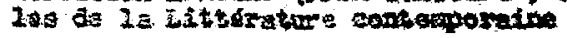

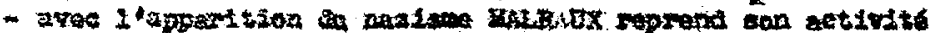

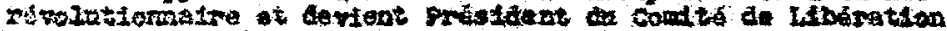

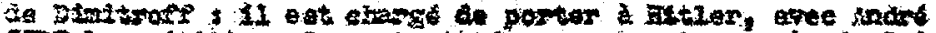

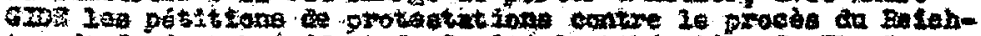
to

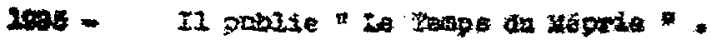

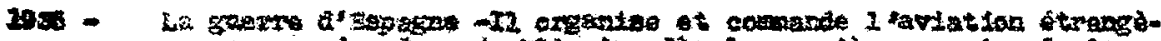

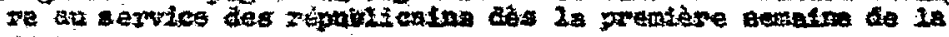
grater.

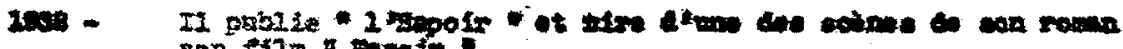

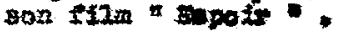

1909

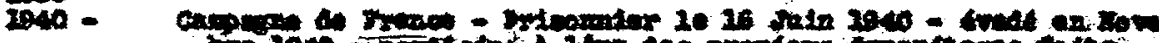

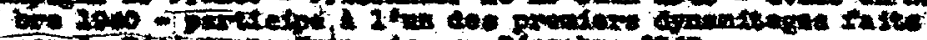

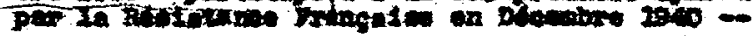

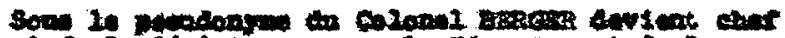

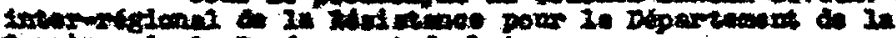

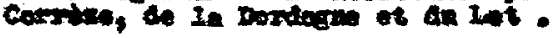

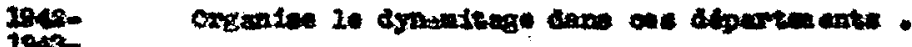

100

$\operatorname{men}$

1040.

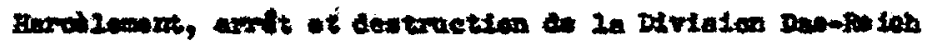

andest

2004 .

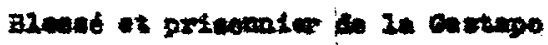

netis

Io4

Disives a Tombtape.

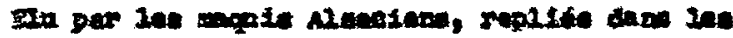

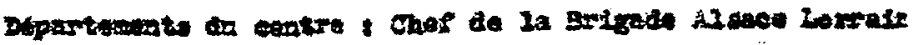

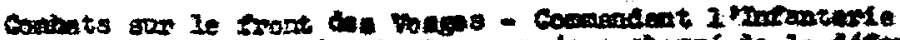

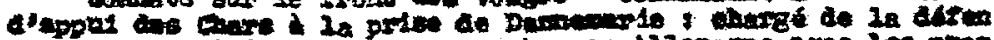

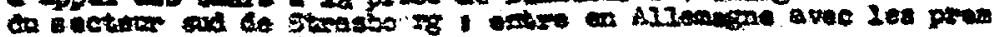

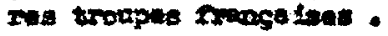

$$
-t-1-3-t-1=
$$

